\title{
Adaptive Medical Image Fusion Method based on NSCT and Unit-Linking PCNN
}

\author{
Wei Liu \\ Zhejiang Gong Shang University, Hangzhou, 310018, China \\ liuxinchen22@163.com
}

\begin{abstract}
This paper presents a self-adaptive medical image fusion method based on combining NSCT and Unit-Linking PCNN together. Firstly, for the strictly registered image to be fused, it will be decomposed at different directions and scales by utilizing nonsubsampled Contourlet transform (NSCT) to obtain low frequency sub-band coefficients and different directions of the high frequency sub-band coefficients. The selected value coefficient of the low frequency sub-band based on edges and high frequency sub-band coefficients are used as external excitation input for Unit-Linking PCNN, fuse low-frequency sub-band according to the first ignition timing of the corresponding point; Taking Canny operator to do the edge detection of the high frequency sub-band, then to fuse it by the first ignition timing of the corresponding point and the results of edge detection. Finally, fusion image will be obtained by NSCT's inverse transformation. The above fusion algorithms will be applied to CT/MRT medical image's fusion experiments, and do experimental comparative analysis with other different fusion algorithms. Most experimental results show that this fusion algorithm is obviously better than the comparative method in both subjective and objective evaluations.
\end{abstract}

Keywords: Medical image fusion; Nonsubsampled contourlet transform; Unit-linking PCNN; Canny operator.

\section{Introduction}

Image fusion is an important branch of information fusion, which is the basis for image analysis and processing. It can fuse several sensor images with different imaging mechanism to a more clear, more intelligible and high quality image For example, this paper proposes a fusion algorithm to fusing CT (Computed Tomography) and MRT (Magnetic Resonance Imaging) image, to integrate both advantages, thus providing more accurate and more comprehensive information for medical diagnosis.

PCNN [1] is a neural network model that is proposed by Eckhorn who want to explain the synchronous behavior of neurons associated with characteristics phenomenon which is observed from the experiment of cat's brain's visual cortex. PCNN is widely applied in many areas of image processing.[2] proposed PCNN image fusion algorithm which is based on wavelet transformation, the spatial frequency of wavelet coefficient is considered as linking strength of PCNN neurons, the fusion coefficient will be determined by the ignition map of wavelet coefficient. But because of the limitations of the wavelet analysis, the high dimensional image information can't be described effectively. [3] Put forwarded a fusion algorithm based on contourlet transform and the PCNN model's improvement. Using PCNN to design fusion strategy, which has a higher identification compared to the effect of wavelet fusion. Since the contourlet transform don't have translation invariance, the fused images will emerge false contour. Therefore, in recent years, the method of combination between PCNN and NSCT (Nonsubsampled contourlet) have been introduced into image fusion field. [4] Realizes imaging fusion by combine NSCT with PCNN through regional energy maximum rule, even though it achieved a good fusion effect, the applied PCNN model was complicated and the number of iterations could not be self-adaptive. In the meanwhile, according to the fact that human visual system is sensitive to image edge information, it is not enough just to adopt a single pixel gray value as external incentives for PCNN's neurons [5]. Regarding the existing problem occurred in [4], this paper puts forward a method of self-adaptive image fusion based on the combination of NSCT and Unit-linking PCNN. This method saves a lot of useful information with 
low frequency. The save of high frequency focused on contour information creates a clearer fusion image, finally the self-adaptive iterations can decrease a certain amount of calculation.

\section{Non subsampled Contourlet Transform (NSCT)}

NSCT is the improvement of traditional CT, CT's Laplacian Pyramid and directional Filter Bank(DFB)[6-8] were separately replaced by NSCT's non subsampled pyramid (NSP) and non-subsampled directional Filter Bank(NSDFB).this improvement of NSCT makes energy more concentrated. And it is better to capture the image's geometric structure, thus to avoid ringing artifacts. The image one is the structure diagram that shows decomposition of image by using NSCT from multi-scale and multi-direction (Figure 1).

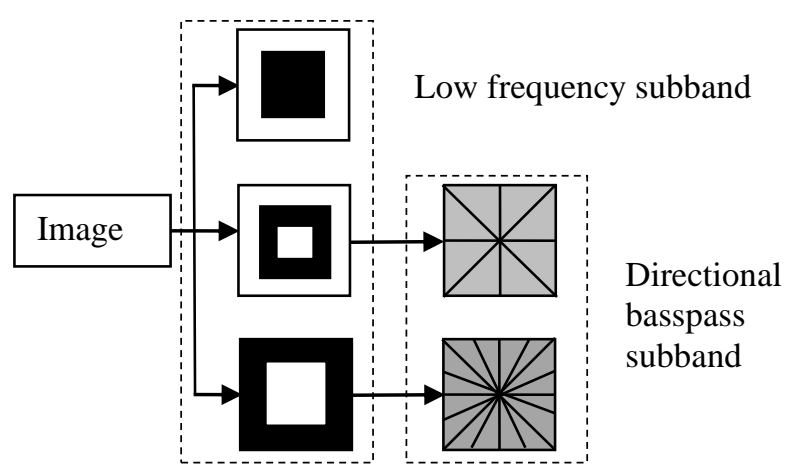

Fig. 1 Structure of nonsubsampled Contourlet transforms

\section{PCNN MODEL(Pulse-coupled neural network model)}

PCNN is a monolayer and feedback-type neural network that is interconnected of a number of neurons. PCNN can extract useful information from complicated backgrounds and don't need learning or training, it has specialties such as sync pulse distribution and global coupling. Its signal form and the processing mechanism are more aligned with physiological basis of human visual neural system. Each of its neurons is composed by three parts: receiving part, modulation part and pulse generating part. This paper proposed that using first ignition time [9] to guide the selection of fusion coefficient and with the help of timing matrix [10] to realize Unit-Linking PCNN iterations adaptation based on Unit-Linking PCNN model [11]. The Unit-Linking PCNN's mathematical formula as per below Eq. (1) (Figure 2).

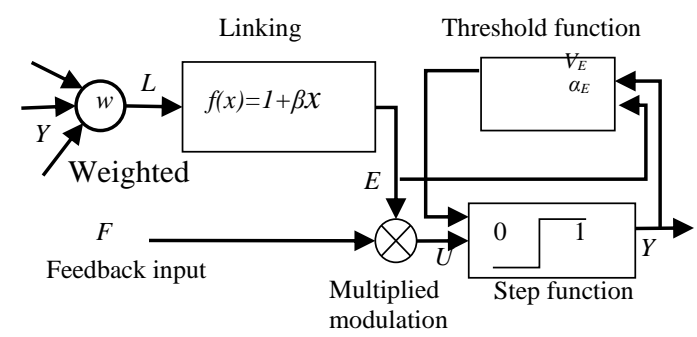

Fig. 2 Structure of PCNN

$$
\left\{\begin{array}{l}
F_{i j}[n]=I_{i j} \\
L_{i j}[n]= \begin{cases}1, & \sum_{K \in N(i, j)} Y_{K}[n-1]>0 \\
0, & \text { otherwize }\end{cases} \\
U_{i j}=F_{i j}[n]\left(1+\beta_{i j} L_{i j}[n]\right) \\
Y_{i j}[n]= \begin{cases}1, & U_{i j}[n]>E_{i j}[n] \\
0, & U_{i j}[n] \leq E_{i j}[n]\end{cases} \\
E_{i j}[n]=\exp \left(-a_{E}\right) E_{i j}[n-1]+V_{E} Y_{i j}[n]
\end{array}\right.
$$




$$
T_{i j}= \begin{cases}n, & \text { first } Y_{i j}[n]=1 \\ 0, & \text { otherwize }\end{cases}
$$

Including: Subscript(i. j) is the label for neuron, $N$ represents the number of Integrations, $F_{i j}(n)$ is feedback input, $\mathrm{I}_{\mathrm{ij}}(\mathrm{n})$ is the external stimulation signal(In the image processing, it show the gray value for picture elements which is located in $(i, j), L_{i j}(n)$ is the input link of neurons, Bij is the linking strength, $U_{i j}(n)$ is the internal action of neurons, $Y_{i j}(n)$ is the (i. j) neuron's output at the $n$ time of interation, $E_{i j}(n)$ and $V_{E}$ are separately representing variable valve output and the threshold value. $\alpha_{E}$ is the time constant for variable threshold function. If $U_{i j}(n)>E_{i j}(n)$, it shows that the neurons produces a pulse, that represents an ignition, $T_{i j}$ is the first ignition time at the point of (i. j). After finishing the PCNN modeling, we can take the characteristics of each neuron output pulse sequence as the significant measure of fusion coefficient, and to decide fusion rule's decision diagram. Because of the faster response of the human eyes to the stronger information, that is to say its sensitive responses to the important features like the edge of the image and texture, (so when) this feature reacts to PCNN model, the element value of neuron is greater, the ignition frequency of this neuron is higher, and the time of opening distributed pulse is earlier. Thus, the ignition time can response the strength of the external input signal to neuron network, so this paper will regard it as the fusion rule's judgment basis.

\section{Image fusion algorithm based on combination of NSCT and Unit-Linking PCNN}

The fusion algorithm:

Through NSCT's multi-scale decomposition to two registered medical source images to get the low frequency sub-band coefficients and high frequency sub-band coefficients, then analyzing the characteristics of each sub-band and designing the corresponding fusion rule respectively. Finally, applying NSCT's inverse transformation to the fused high and low frequency coefficients, to obtain reconstructed image.

\subsection{Low frequency sub-band coefficients fusion rules}

When processing low-frequency sub-band coefficients with PCNN, we usually take low frequency sub-band coefficients as PCNN's external drive directly. But, directly using low-frequency sub-band coefficients as PCNN's external drive will make people ignore the low frequency information and it will affect the image visual effect.

In order to overcome the shortcoming above, in view of the low frequency sub-band contains lots of useful information in the image, and concentration of the image's main energy, thus this paper chooses edge selection value[12] as PCNN's external stimulus. Edge selection value E reflects that the edge of the image's information in a horizontal, vertical and diagonal direction better reserve the original image details.

The mathematical definition based on the edge of the variable $\mathrm{E}$ is like below

$$
\begin{aligned}
& E_{A}(i, j)=\left(F_{1} * C_{n_{0}}^{A}\right)^{2}(i, j)+\left(F_{2} * C_{n_{0}}^{A}\right)^{2}(i, j)+\left(F_{3} * C_{n_{0}}^{A}\right)^{2}(i, j) \\
& E_{B}(i, j)=\left(F_{1} * C_{n_{0}}^{A}\right)^{2}(i, j)+\left(F_{2} * C_{n_{0}}^{A}\right)^{2}(i, j)+\left(F_{3} * C_{n_{0}}^{A}\right)^{2}(i, j)
\end{aligned}
$$

Among “*" represents convolution

$$
F_{1}=\left[\begin{array}{ccc}
-1 & -1 & -1 \\
2 & 2 & 2 \\
-1 & -1 & -1
\end{array}\right], F_{2}=\left[\begin{array}{ccc}
-1 & 2 & -1 \\
-1 & 2 & -1 \\
-1 & 2 & -1
\end{array}\right], F_{3}=\left[\begin{array}{ccc}
-1 & 0 & -1 \\
0 & 4 & 0 \\
-1 & 0 & -1
\end{array}\right]
$$

The concrete steps of Low frequency sub-band fusion is like below:

Firstly, using NSCT to decompose the original image A (figure CT) and the original image B (figure MRI), and to respectively obtain low frequency sub-band coefficients $C_{n_{0}}^{A l}(i, j)$ and $C_{n_{0}}^{B l}(i, j)$ for original A and original B.

Secondly, using formula 5 and formula 6 to respectively calculate original image A's and original image B's variable choice E based on the edge at the point (i. j); then taking E as Eq. (1)'s excitatory input, through Eq. (2) to obtain both original image A's and B's time matrix TA and TB by the end of the iteration. 
Finally, through Eq. (6) and Eq. (7) to judge and choose the low frequency sub-band $C_{n_{0}}^{F l}(i, j)$

$C_{n_{0}}^{F l}(i, j)= \begin{cases}C_{n_{0}}^{A l}(i, j) & T_{n_{0}}^{A}(i, j)<T_{n_{0}}^{B}(i, j) \\ C_{n_{0}}^{B l}(i, j) & T_{n_{0}}^{A}(i, j)>T_{n_{0}}^{B}(i, j)\end{cases}$

Ignition timing are the same, select $\mathrm{E}$ largest fusion rule.

$C_{n_{0}}^{F l}(i, j)= \begin{cases}C_{n_{0}}^{A l}(i, j) & E_{A}(i, j)>E_{B}(i, j) \\ C_{n_{0}}^{B l}(i, j) & E_{A}(i, j)<E_{B}(i, j)\end{cases}$

\subsection{The fusion rules for High frequency sub-band}

For the high frequency sub-band, because the human vision is mainly sensitive to image's edge details, so we detect the high frequency coefficients located in edges of images. Using edge detection operator to get high frequency sub-band coefficients transformed by NSCT. Canny [13] edge detection was carried out on the high frequency sub-band edge detection in this paper. Then the high frequency sub-band coefficients will be used to iterate as the PCNN external incentive, when the iteration finished, taking the ignition time as the basis of the coefficient of edge fusion choice.

The specific steps for high frequency sub-band fusion are as follows

Firstly, The source image A and B get the high frequency coefficients $C_{n, k}^{A h}(i, j)$ and $C_{n, k}^{B h}(i, j)$ at the direction of $\mathrm{K}$ on the scale of $\mathrm{N}$ through NSCT decomposition, canny edge detection is carried out on the high frequency subband edge detection, then getting two edges matrix $\mathrm{D}_{\mathrm{A}}$ and $\mathrm{D}_{\mathrm{B}}$.

Then, through the formula $\mathrm{A}$ and $\mathrm{B}$ to do $\mathrm{N}$ times iteration, and getting two time matrix $\mathrm{Ta}$ and $\mathrm{Tb}$ after finishing.

Lastly, judging and selecting new high frequency sub-band $C_{n, k}^{F h}(i, j)$ through the Eqs. $(8-10)$

When one of two images' high frequency coefficients is situated in the edge of the image, refer to the Eq. (8) to doing selection:

$$
C_{n, k}^{F h}(i, j)= \begin{cases}C_{n, k}^{A h}(i, j) & D_{n, k}^{A h}(i, j)=1 \text { and } D_{n, k}^{B h}(i, j)=0 \\ C_{n, k}^{B h}(i, j) & D_{n, k}^{A h}(i, j)=0 \text { and } D_{n, k}^{B h}(i, j)=1\end{cases}
$$

When both of the two images' high frequency coefficients are situated in the edge of the image, using the weighted average rule when doing the fusion for this position's coefficient, then selecting refer to the Eq. (9)

$$
C_{n, k}^{F h}(i, j)=\frac{1}{2}\left[C_{n, k}^{A h}(i, j)+C_{n, k}^{B h}(i, j)\right]
$$

When both of the two images' high frequency coefficients aren't situated in the edge of the image, using the earliest principles of ignition when doing the fusion for this position's coefficient.

$$
C_{n, k}^{F h}(i, j)= \begin{cases}C_{n, k}^{A h}(i, j) & T_{n, k}^{A h}(i, j)<T_{n, k}^{B h}(i, j) \\ C_{n, k}^{B h}(i, j) & T_{n, k}^{A h}(i, j)>T_{n, k}^{B h}(i, j)\end{cases}
$$

\section{Experiments and result analysis}

In order to verify the validity of the algorithm in this paper, under the environment of MATLAB2010, doing simulation respectively for three groups of gray CT/MRT images and two groups of color SPECT/MRI images. Normal brain's CT and MRI, is like the figure 3a and b; Multiple cerebral infarction's CT and MRI, is like the figure 4a and b; Brain stroke combine's CT and MRI, is like the figure 4a and b; the brain's SPECT and MRI, is like 9a and b; The normal brain's SPECT and MRI, is like the figure 4a and b; The scale decomposition filter of the NSCT in the experiment uses"9-7" filter, the direction decomposition filter uses "dmaxflat7" filter, decom position dimensions are all 4 layers, scales decompose into 4, 4, 8, 16 from coarse to fine direction. The related parameters setting for PCNN model like below: $\alpha_{\mathrm{E}}=0.2, \mathrm{~V}_{\mathrm{E}}=20$ Number of iterations $\mathrm{N}=200$.It is impossible that every neuron keep the same link intensity $\beta$ in the human visual system, the clarity is image edge's sharpness, it is a physical quantity to describe the microscopic image ability. To adaptively set the $\beta$, this paper takes the clarity as the value of the link intensity for corresponding neurons. 
The mathematical definition for Pixels (i. j)'s clarity [14] is as follows

$\sum_{(x, y) \in W}\left\{[f(x, y)-f(x+1, y)]^{2}+[f(x, y)-f(x, y+1)]^{2}\right\}$

w is the $3 * 3$ 's window centered (i. j).

\subsection{Fusion of CT/MRI grayscale images}

This paper selects other three different fusion algorithms to be compared with the fusion algorithm proposed by this paper. The fusion image Figure 3 (c) and Figure 4 (c) are obtained by using wavelet decomposition proposed by [2] and taking wavelet coefficients with more ignition as the corresponding fusion coefficient, the fusion image Figure 3 (d) and Figure 4 (d) are obtained by using the method of Contourlet transformation and IPCNN combined proposed by [3]. Figure 3 (e) and Figure 4 (e), respectively, from [15] and the fusion method NSCT PCNN combining the low frequency section uses a method based on the edge of the high-frequency portion of coefficients and Improvement the PCNN combined fusion image fusion frequency portion derived. Figure 3 (e) and Figure 4 (e), Figure 3 (f) and Figure 4 (f) are the fusion results by referring to the fusion algorithm that this paper proposed.

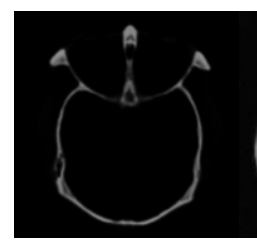

(a)

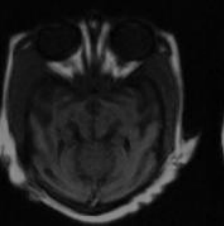

(b)

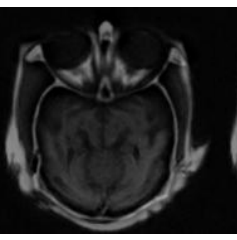

(c)

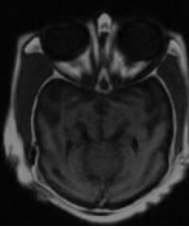

(d)

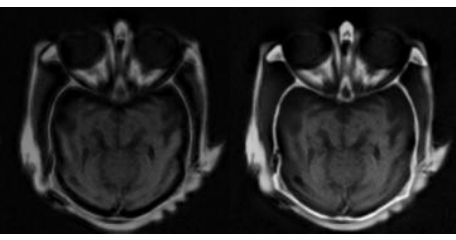

(e) (f)

Fig. 3 Fusion results for a normal persons' brain CT/MRI medical images: a, CT; b, MRI; c, WT+PCNN; d, Contourlet+pcnn; e, NSCT+PCNN; f, Method of this paper.

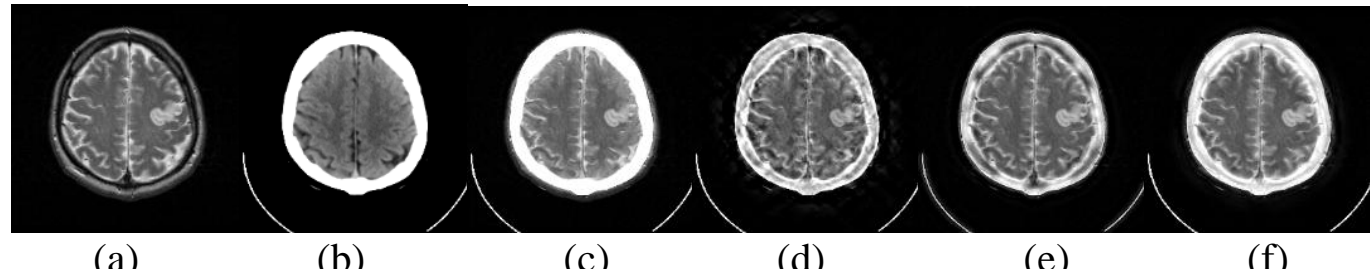

Fig. 4 Fusion results for a diseased persons' with acute stroke CT/MRI medical images: a, CT; b, MRI; c, WT+PCNN; d, Contourlet+pcnn; e, NSCT+PCNN; f, Method of this paper.

\subsection{Simulation experiment and results analysis}

From Fig. (3) to fig. (4) we can see: wavelet transform makes fusion image distortion, and having a jagged border, the caused reason is that the wavelet transform has the limitation with lack of translation invariance. For the Contourlet transform, it improved the image edge distortion to some degree; but Contourlet transform will cause spectrum aliasing phenomenon, and the fusion image has obvious false contour phenomenon, the experiment results show that NSCT transform obviously improved fusion image's false contour phenomenon. And From the experimental results can be seen in the fused image obtained by fusion methods NSCT and PCNN combining fusion image fusion method herein in detail, brightness, or have better clarity, while less amount of calculation at the same time.

\subsection{Evaluation index for fusion image}

The above has carried on qualitative analysis of this paper proposed algorithm in this paper and other several algorithms. Now carrying on quantitative comparison for above three algorithms through information entropy [16] (IE), mutual information [16] (MI), mean gradient [16] (MG) and standard deviation [16] (SD) objective evaluation index etc.

Fused image corresponding evaluation are shown in Table 1, Table 2 below. 
Table1 The evaluation index for Normal brain CT/MRI different fusion method

\begin{tabular}{ccccc}
\hline Evaluation index & WT+PCNN & Contourlet+PCNN & NSCT+PCNN & Method of this paper \\
\hline IE & 6.0434 & 6.2891 & 6.4312 & $\mathbf{6 . 5 8 6 1}$ \\
MI & 0.5791 & 0.6635 & 0.6635 & $\mathbf{0 . 6 8 3 2}$ \\
AG & 4.1043 & 4.1745 & 4.2017 & $\mathbf{4 . 2 8 2 7}$ \\
SD & 35.667 & 37.463 & 41.689 & $\mathbf{4 3 . 8 9 4}$ \\
\hline
\end{tabular}

Table2 The evaluation index for cerebral stroke CT/MRI different fusion method

\begin{tabular}{ccccc}
\hline Evaluation index & WT+PCNN & Contourlet+PCNN & NSCT+PCNN & Method of this paper \\
\hline IE & 5.3144 & 5.3992 & 5.5241 & $\mathbf{5 . 6 8 8 6}$ \\
MI & 0.5734 & 0.5971 & 0.6211 & $\mathbf{0 . 6 7 4 5}$ \\
AG & 5.6221 & 5.6612 & 5.7531 & $\mathbf{5 . 9 7 6 3}$ \\
SD & 80.467 & 81.184 & 81.743 & $\mathbf{8 2 . 5 4 1}$ \\
\hline
\end{tabular}

Seen from the values of two tables, our proposed method is better than other different method in objective evaluation, it is consistent with visual judgment result.

\section{Conclusion}

This paper presented self-adaptive medical image fusion method based on Combining NSCT and Unit-Linking PCNN together, at last successfully applying it into the fusion of gray CT/MRI images and color SPECT/MRI images. The results of theory analysis and simulation experiments showed that the paper proposed algorithm can get a better fusion effect than comparison algorithm, it's able to preserve the important objective characteristics of CT images and MRT images, it showed the feasibility, effectiveness and adaptability of this algorithm when it's applied to the fusion of CT images and MRI images.

\section{Acknowledgements}

National Natural Science Foundation (NO:61374022).

\section{References}

[1] Eckhorn R, Reitboeck H J, Arndt M, et al. Feature linking via synchronization among distributed assemblies: Simulations ofresults from cat visual cortex [J]. Neural Computation (S0899-7667), 1990, 2(3): 293-307.

[2] Xue Shizhong, Zhou Aiping, Liang Jiuzhen. PCNN multi-sensor image fusion based on wavelet transform [j]. Computer Engineering and Applications, 35,2011; 210-213

[3] Liu Shengpeng, Fang Fong. The fusion algorithm based on Contourlet transform and IPCNN and its application in visible light and infrared image fusion. J Infrared Millim Waves, 2007, 26 (3); 217-221.

[4] Wen Liming, Peng Li, Xu Hong. Remote sensing image fusion algorithm based on NSCT and PCNN. Computer Engineering. 2012, 11(11); 196-198

[5] Qu Xiaobo, Yan Jingwen (University X, Xiamen, et al. Image Fusion Algorithm Based on Spatial Frequency-Motivated Pulse Coupled Neural Networks in Nonsubsampled Contourlet Transform Domain [J]. Acta Automatica Sinica, 2008, 34(12):1508-1514.

[6] Yang Yong, Zheng Wenjuan, Sue Huang, et human visual perception characteristics nonsubsampled Contourlet transform domain multi-focus image fusion [J]. Chinese Journal of Image and Graphics, 2014, 19 (3).

[7] Shen Yu, Ren Enen, Dang Jian-Wu. A nonsubsampled contourlet transform based medical image fusion method [J]. Information Technology Journal, 2013, 12 (4):749-755. 
[8] Ye Chuanqi, Zhang Liwen, Zhang Zhiyong. SAR and panchromatic image fusion based on rregion features in nonsubsampled contourlet transform domain [J]. IEEE International Conference on Automation and Logistics, ICAL, 2012, 2012, 14(8):358-362.

[9] Liu Qing, Ma Yi one kind of new de-noising algorithm [J] when PCNN matrix forming an image based on Electronics and Information Technology, 2008, 30: 1869-1873.

[10]Kong Weiwei, Lei Yang NSCT based domain I 2CM image fusion method [J]. Systems Engineering Theory and Practice, 2012, 11 (11): 2557-2563.

[11] Gu Xiaodong, Zhang Liming, Yu Daoheng. With no need to select the parameters of Unit-linking PCNN automatic image segmentation [J]. Circuits and Systems, 2007, 12 (6): 54-59.

[12]Chao Rui, Zhang Ke, Li Yanjun fusion algorithm [J] Journal of the image based on wavelet transform electronics, 2004,32 (5): 750-753.

[13] Wang Zhi, Saixian an adaptive Canny edge detection method based on the theory [J] Chinese Journal of Image and Graphics, 2004, 9 (8): 957-962.

[14]Zhang Wei,Cao Lelin, Hou Chingyu, et other multi-band infrared point target detection algorithm RX performance analysis [J] Photonica Sinica, 2009,38 (7): 1757-1761.

[15]Li Meili, Li Yanjun, Wang Hongmei, etc. based on infrared and visible image fusion method NSCT and PCNN of [J] Optical Engineering, 2010, section 6 (6): 90-95.

[16] Hu Liangmei, Gao Jun, He Kefeng image fusion quality assessment methods [J]. Journal of Electronics, 2014,32 (12A): 218-221 